\title{
面向流场可视化的沉浸式虚拟现实协作框架
}

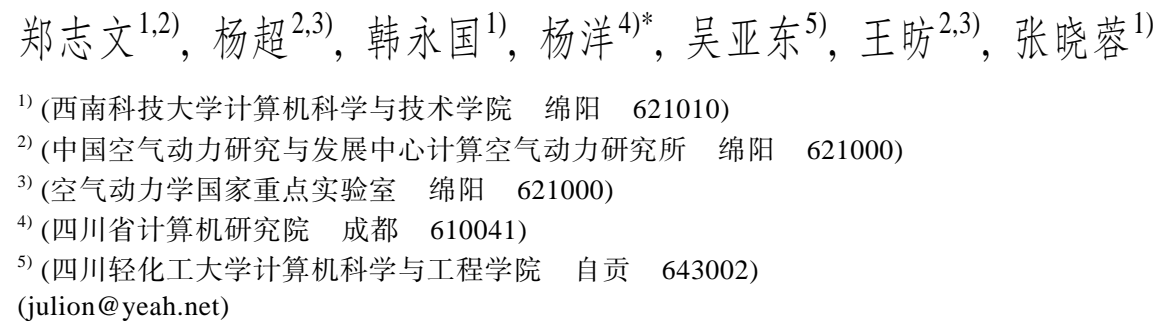

\section{Immersive Virtual Reality Collaboration Framework for Flow Field Visualization}

\author{
Zheng Zhiwen ${ }^{1,2)}$, Yang Chao ${ }^{2,3)}$, Han Yongguo ${ }^{1)}$, Yang Yang ${ }^{4)^{*}}$, Wu Yadong ${ }^{5)}$, Wang Fang ${ }^{2,3)}$, and \\ Zhang Xiaorong ${ }^{1)}$ \\ 1) (School of Computer Science and Technology, Southwest University of Science and Technology, Mianyang 621010) \\ ${ }^{2)}$ (Computational Aerodynamics Institute, China Aerodynamics Research and Development Center, Mianyang 621000) \\ 3) (State Key Laboratory of Aerodynamics, Mianyang 621000) \\ 4) (Sichuan Institute of Computer Research, Chengdu 610041) \\ 5) (School of Computer Science and Engineering, Sichuan University of Science and Engineering, Zigong 643002)
}

\begin{abstract}
Aiming at the problems of the single collaboration mode and the high cost of building a collaboration environment of the existing flow field collaboration analysis application, according to the requirements of flow field collaboration analysis, an immersive virtual reality collaboration framework for flow field visualization is proposed. Firstly, the collaboration message that supports flow field collaboration synchronization is defined. Then a collaborative pipeline tree is proposed that supports the sharing of flow field visualization results. Finally the demonstration collaboration analysis mode and free collaboration analysis mode of immersive virtual reality flow field visualization collaborative analysis are designed and implemented. Users can choose the appropriate collaboration analysis mode according to the actual task, to sup-
\end{abstract}

收稿日期：2021-07-06; 修回日期：2021-09-08. 基金项目：国家自然科学基金(61872304，61802320); 国家数值风洞工程 (NNW2019ZT6-A17); 国防科技专项(19zg6105). 郑志文(1996-), 男, 硕士研究生, CCF 会员, 主要研究方向为虚拟现实技术、人机 交互; 杨超(1988一), 男, 博士, 高级工程师, 主要研究方向为虚拟现实技术、流场可视化; 韩永国(1963一), 男, 博士, 教授, 硕士生 导师, CCF 会员, 主要研究方向为可视化与可视分析、人机交互; 杨洋(1982一), 男, 硕士, 高级工程师, 论文通讯作者, 主要研究方 向为虚拟现实技术、人机交互; 吴亚东(1979-), 男, 博士, 教授, 博士生导师, CCF 会员, 主要研究方向为可视化与可视分析、人机 交互; 王昉(1976一), 男, 博士, 高级工程师, CCF 会员, 主要研究方向为科学可视化、高性能计算; 张晓蓉(1975一), 女, 硕士, 讲师, 主要研究方向为可视化与可视分析、人机交互. 
port the team in the flow field collaboration analysis. By building a prototype system and performing a demonstration collaboration analysis and a free collaboration analysis on the flow field data respectively, from the perspective of observation of different users, the collaboration process of the two collaborative analysis modes is demonstrated.

Key words: immersive analysis; virtual reality; flow field visualization; collaborative analysis

流场可视化是科学可视化的重要分支之一，它 使用计算机图形学方法将流场数据进行绘制、渲 染，并转化为直观的图形图像，通过人机交互手段 方便科学家直观、形象、高效地分析和理解流场的 物理现象, 洞察流场数据中蕴含的复杂物理规律. 在流场数据中往往包含了速度、压强和温度等多方 面的流场信息，而且不同空间区域的流场特征、规 律也存在差异, 为了提高流场可视化分析效率, 在 对大规模复杂流场数据进行可视分析时往往需要 进行团队协作 ${ }^{[1]}$. 近年来, 随着计算流体动力学 (computational fluid dynamics, CFD) 和数值模拟技 术的快速发展，数值计算产生的流场数据规模和 复杂度都不断增加, 流场可视化协作分析变得尤 为重要.

随着一系列高分辨率的头戴式显示器 (head mounted display, HMD)(如 Oculus Rift, HTC Vive) 的出现, 沉浸式虚拟现实环境的搭建难度和成本 大大降低，相关技术开始广泛应用于军事、工程、 教育、生物和医学等众多领域. 将沉浸式虚拟现实 技术应用到科学可视化 ${ }^{[2]}$ 中, 通过直观的立体成 像、三维交互、立体听觉和三维触觉反馈等方法, 拓展了人机交互的手段, 使用户与数据的交互更 加自然、和谐, 可帮助用户高效地理解大规模复杂 流场数据蕴含的空间位置关系. 沉浸式虚拟现实 技术 ${ }^{[3]}$ 还支持高效的团队协作，其团队协作质量与 面对面和视频会议相比，几乎没有任何区别.

沉浸式虚拟现实协作在许多方面已取得了一 定的研究成果, 但是相关系统多数是依据各自领 域的需求开发的, 采用的可视化引擎大多数是游 戏引擎 ${ }^{[4-6]}$, 其在流场数据的计算、绘制和表达方 面, 效率远低于传统的可视化框架 ${ }^{[7]}$, 无法满足实 时流场可视化协作分析的需求. 现有的流场可视 化软件(如 ParaView, Ensight 等)虽然支持远程协 作，但是其协作模式单一，只支持主客户端与数据 进行交互式分析、其余客户端观察的协作模式，并 且可视化结果通过二维图像呈现, 丢失了三维深 度信息, 不能满足专家实时协作分析的需求. 还有 一些基于洞穴状自动虚拟环境(cave automatic virtual environment, CAVE)搭建的沉浸式虚拟现实流 场可视化协作分析系统 ${ }^{[8]}$, 不仅搭建时间和成本耗 费巨大, 而且还可能存在远程专家派遣到本地进 行协作的时间和成本问题 ${ }^{[9]}$.

针对上述问题，本文基于沉浸式虚拟现实交 互系统 ${ }^{[7]}$ 和流场协作分析需求, 设计了面向流场可 视化的沉浸式虚拟现实协作框架; 提出了支持多 用户流场可视化算法结果共享的协作管线树; 并 定义了沉浸式虚拟现实流场可视化协作消息; 提 出了适用于流场可视化协作的演示协作模式和自 由协作模式, 并将 2 种协作分析模式应用在沉浸式 虚拟现实流场协作分析中.

\section{1 相关工作}

\section{1 虚拟现实流场可视化技术}

20 世纪 90 年代初, 虚拟现实技术已开始融人 流场可视化的研究. Hibbard 等 ${ }^{[10]}$ 提出开源三维流 场可视化系统，支持基于 Widget 的用户界面和快 速的可视化反馈, 允许地球科学家交互式地探索 庞大而复杂的数据集. Bryson 等 ${ }^{[11]}$ 构建的虚拟风 洞系统可在三维非定常流场中绘制流线, 以观察 粒子的运动规律. Wesche $\mathrm{e}^{[12]}$ 结合虚拟现实技术在 工作台上的虚拟汽车周围显示流线, 允许用户通 过手势和按钮调整流线参数, 以评估汽车的气动 性能. Kageyama 等 ${ }^{[13]}$ 提出的三维矢量场可视化系 统, 允许仿真研究人员交互式可视化流线、示踪粒 子运动和等值面. Wasfy 等 ${ }^{[14]}$ 在沉浸式虚拟现实环 境中实现了 CFD 结果的可视化, 并支持呈现多种 可视化算法.

虚拟现实技术与计算机硬件的发展为虚拟现 实在流场可视化领域的广泛应用带来了机遇. ParaView ${ }^{[15]}$ 允许用户通过 HMD 将桌面端可视化结 果呈现在沉浸式虚拟现实环境中. Dhillon 等 ${ }^{[16]}$ 在 虚拟现实环境下实现了仿生俯仰板的浴尾流可视 化, 支持用户分析尾流中漩涡的形成和脱落以及 
特定时间内的流量参数关系. O’Leary 等 ${ }^{[17]}$ 在可视 化工具库(visualization toolkit, VTK)的基础上，提 出了支持 HMD 的可视化方法, 使 VTK 具备沉浸 式虚拟现实功能, 帮助研究人员进行沉浸式虚拟 现实科学可视化研究. $\mathrm{Lu}$ 等 ${ }^{[18]}$ 设计了基于虚拟现 实和手势交互技术的气候矢量场数据感知系统, 帮助用户更有效地理解和分析数据. 陈呈等 ${ }^{[19]}$ 提 出的流场可视化系统结合了手势和凝视 2 种交互 通道，支持多视图、多角度在沉浸式虚拟现实环境 下探测流场. 但是, 目前的虚拟现实流场可视化研 究都局限于仅为单个用户提供高水平的多感官沉 浸感，对于多人协作的沉浸式虚拟现实流场可视 化研究甚是稀缺.

\section{2 沉浸式虚拟现实协作技术}

沉浸式虚拟现实协作技术是指多个用户在同 一个沉浸式虚拟现实环境中进行交互并完成协作 任务. 它是一个令人兴奋的新领域, 在改进团队解 决问题的方式方面具有巨大潜力，人们对它的关 注度日益渐增. Wheless 等 ${ }^{[20]}$ 提出的沉浸式虚拟现 实协作系统, 允许多个用户共同可视化、讨论并与 环境中的数据集交互. Imai 等 ${ }^{[21]}$ 构建的虚拟现实 协作系统允许用户在虚拟现实环境中记录语音信 息以及虚拟化身的身体动作和手势, 以便其他用 户稍后回放. Jackson 等 ${ }^{[22]}$ 在学校课程教学中融人 沉浸式虚拟现实协作技术, 可以帮助学生更好地 学习和理解知识. Kaiser 等 ${ }^{[23]}$ 在矿山设计和规划中 使用沉浸式虚拟现实协作技术, 使研究人员更好 地理解数据的空间关系, 提高采矿决策的正确性. Kockro 等 ${ }^{[24]}$ 提出支持医学影像数据协作分析的沉 浸式虚拟现实系统, 允许用户在虚拟环境中使用 手持设备直观地与复杂的三维数据交互，同时与 更多的用户共享这些信息. Sharma 等 ${ }^{[25]}$ 提出用于 疏散演习中灾害准备和响应的沉浸式虚拟现实协 作系统, 消除了参与者受伤的风险. Cordeil 等 ${ }^{[26]}$ 在 面对面 CAVE 和远程 HMD 中分别进行了协作分析 任务, 实验结果表明, 在远程 HMD 条件下的搜索 任务不仅与在 CAVE 条件下一样精确, 而且完成 得更快. Lee 等 ${ }^{[5]}$ 提出的沉浸式虚拟现实协作系统, 支持同一地点的多个用户进人沉浸式虚拟现实环 境, 允许用户在虚拟现实环境的任何地方自由地 放置创作界面和可视化结果，同时支持用户单独 或协作完成数据分析任务.

由于流场数据结构复杂、数据点数量众多, 单 个用户进行流场数据分析的质量和效率不容乐观, 往往需要多个研究人员共同提供新的思路和解决
方案, 以提高流场分析结果的正确性, 这使得研究 沉浸式虚拟现实流场可视化协作迫在眉睫. 当前, 专门针对流场可视化的沉浸式虚拟现实协作研究 还比较匮乏, 虽然在相关的研究已有一定成果, 但 其渲染引擎不能满足流场可视化的要求, 已有的 协作模式和搭建成本昂贵的沉浸式虚拟现实协作 环境并不适用于流场可视化协作研究. 因此, 本文 提出了适用于流场可视化的沉浸式虚拟现实协作 框架，提出了支持可视化结果共享的协作管线树; 设计了支持不同流场协作分析任务的演示协作分 析模式和自由协作分析模式, 允许多个用户在沉 浸式虚拟现实环境中进行流场可视化协作分析.

\section{2 本文框架}

\section{1 设计需求}

在沉浸式虚拟现实流场可视化协作分析时, 研究人员在现实环境中无论是处于同一物理位置, 还是彼此处于远程异地, 都希望在沉浸式虚拟环 境中彼此能够如同面对面, 置身于同一流场环境 内. 另外, 在研究人员对流场数据进行分析时, 既 希望能够独立地专注于自身擅长或重点关注的内 容, 又希望能够随时将分析过程及分析结果共享 给团队其他成员. 因此, 本文认为沉浸式虚拟现实 流场可视化协作框架应当满足以下设计需求.

（1）能够为团队的研究人员构建面对面的同 一虚拟现实流场环境.

(2) 具有流场数据交互的彼此可见能力, 以方 便研究人员更好地理解可视化分析结果.

（3）支持便捷、高效地查看流场可视化分析结 果的共享环境.

(4) 为研究人员提供相对自由、独立的虚拟现 实流场可视分析环境.

\section{2 框架设计}

为了满足沉浸式虚拟现实流场可视化协作的 设计需求，本文提出了图 1 所示面向流场可视化的 沉浸式虚拟现实协作框架，它由房间管理、用户身 份、协作管线树、协作分析模式和协作消息 5 个部 分组成.

\subsection{1 房间管理}

在沉浸式虚拟现实协作中, 多个协作团队位 于同一沉浸式虚拟现实环境中, 由于团队之间的 协作任务和参与人员之间的协作分析模式不相同, 在执行协作任务的过程中, 不同团队的人员相互 干扰, 影响协作任务的完成. 


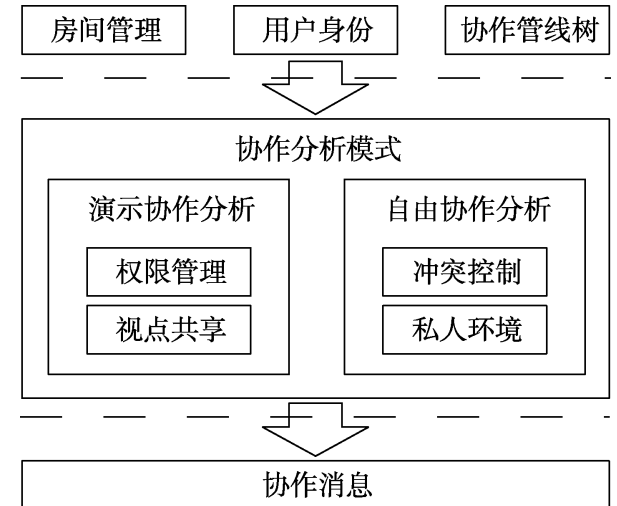

图 1 本文框架

因此, 为了提供多个协作团队能够同时进行 协作分析的环境，本文构建了支持不同协作分析 模式的沉浸式虚拟现实协作房间. 不同房间的协 作分析模式可以分为演示协作分析模式和自由协 作分析模式 2 类，以支持多个团队在沉浸式虚拟现 实环境下进行协作分析.

\subsection{2 用户身份}

在协作团队中，每个成员都有各自的身份，其 根据分析任务、职责等来定义，用户身份决定了用 户权限. 在流场数据自由探索过程中, 多个用户承 担着相应的分析任务, 需要使用不同可视化算法 进行数据分析, 但在沉浸式虚拟现实环境下可视 化结果的呈现需要一定的时间，在这段时间内不 能被其他用户的可视化算法渲染影响，否则就会 中断团队协作分析任务的执行. 因此, 本文通过可 执行的交互操作将用户身份划分为特权用户和普 通用户，可在一定程度上避免多用户交互冲突产 生而影响协作任务的执行. 如表 1 所示, 用户各自 拥有不同的权限，特权用户占主导地位，拥有对流 场数据的所有权, 允许根据需求选择相应的流场 可视化算法进行交互式可视化探索分析; 而普通 用户只能进行观察，为了防止虚拟界面遮挡和支

表 1 用户权限

\begin{tabular}{ccc}
\hline \multicolumn{1}{c}{ 交互操作 } & 特权用户 & 普通用户 \\
\hline 界面呼唤 & $\sqrt{ }$ & $\times$ \\
界面点击 & $\sqrt{ }$ & $\times$ \\
界面移动 & $\sqrt{ }$ & $\sqrt{ }$ \\
配置控件呼唤 & $\sqrt{ }$ & $\times$ \\
配置控件抓握 & $\sqrt{ }$ & $\times$ \\
配置控件移动 & $\sqrt{ }$ & $\times$ \\
可视化工具选择 & $\sqrt{ }$ & $\times$ \\
可视化算法更新 & $\sqrt{ }$ & $\times$ \\
空间导航 & $\sqrt{ }$ & $\sqrt{ }$ \\
\hline
\end{tabular}

持调整观察角度, 赋予其对界面的移动和空间导 航交互权限, 以便理解特权用户的分析方法和结 果. 此外, 在不同的协作分析模式中, 特权用户和 普通用户的数量限制不同.

另外，在沉浸式虚拟现实协作环境中，远程用 户通常由图形角色或化身表示, 其首要任务是提 供非语言交流线索以表示精确的物体操作 ${ }^{[27]}$ 和运 动任务 ${ }^{[28]}$, 这给用户和合作者创造了一种存在于 虚拟环境中并与伙伴共同存在的感觉，能够增强 用户在协作任务中的参与感和存在感. 因此, 本文 为每个用户构建了一个如图 2 所示由透明圆球和 射线组成的虚拟化身. 在沉浸式虚拟现实协作环 境中，虚拟化身脸部方向表示用户的实际脸部方 向, 透明圆球的位置表示用户的空间位置, 射线表 示用户的凝视方向和位置. 同时, 为防止遮挡可视 化结果，支持用户隐藏虚拟化身，只保留其对配置 控件和数据的交互共享协作信息.

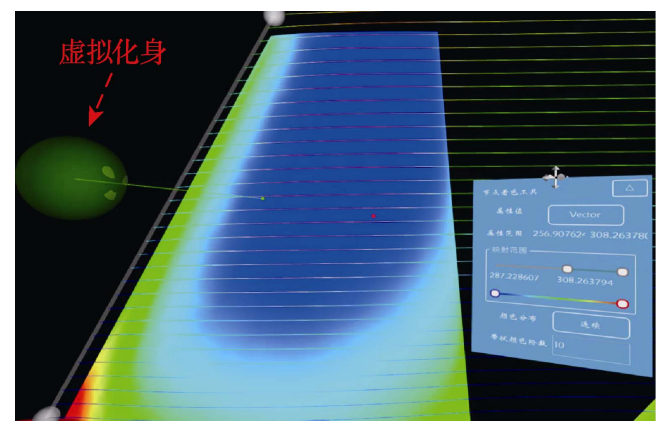

图 2 虚拟化身

\subsection{3 协作管线树}

在对数据进行分析时，多个用户通过多种可 视化算法呈现数据的多种物理量. 多种可视化算 法都是以原数据为根节点进行拓展, 产生更多可 视化算法节点. 在可视化算法节点数量增加关系 复杂时, 传统桌面的呈现方式难以直观地展示算 法节点与多个用户之间的关联关系. 因此, 在基于 可视化算法树的可视化网络管理方法 ${ }^{[7]}$ 基础上, 本 文提出了一种如图 3 所示支持多用户协作共享的 协作管线树. 其中，可视化算法节点的颜色与用户 的虚拟化身对应，以隐喻节点与用户之间的关系;

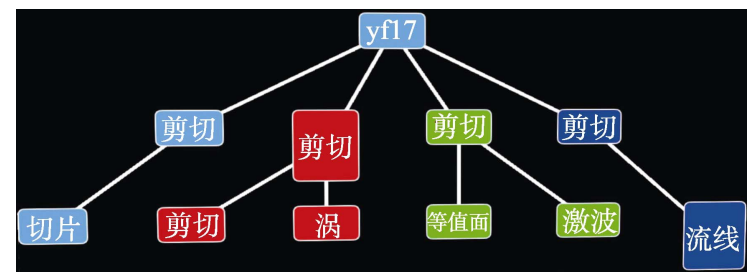

图 3 协作管线树 
节点高度表示状态是否处于共享状态.

在沉浸式虚拟现实协作环境中，协作管线树 变化过程如图 4 所示. 其中, 在各自的剪切可视化 算法节点下，红色用户和绿色用户分别先后添加 并提交了流线和激波可视化算法节点, 协作管线 树上依次新增流线和激波节点, 并通过调整节点 高度把节点状态改为共享状态. 浅蓝色用户在分 别接受流线和激波节点后，协作管线树上对应节 点高度变回初始形态，接着流场数据会执行对应 节点算法并在沉浸式环境中呈现其可视化结果. 绿色用户删除激波节点后，协作管线树会同步删 除, 沉浸式协作环境中也会删除该可视化结果. 另 外，在演示协作模式中，特权用户能够编辑协作管 线树上所有节点，普通用户没有与之交互的权限; 但在自由协作模式中，特权用户只能编辑协作管线 树上自己新增的节点, 并接受共享的可视化节点.
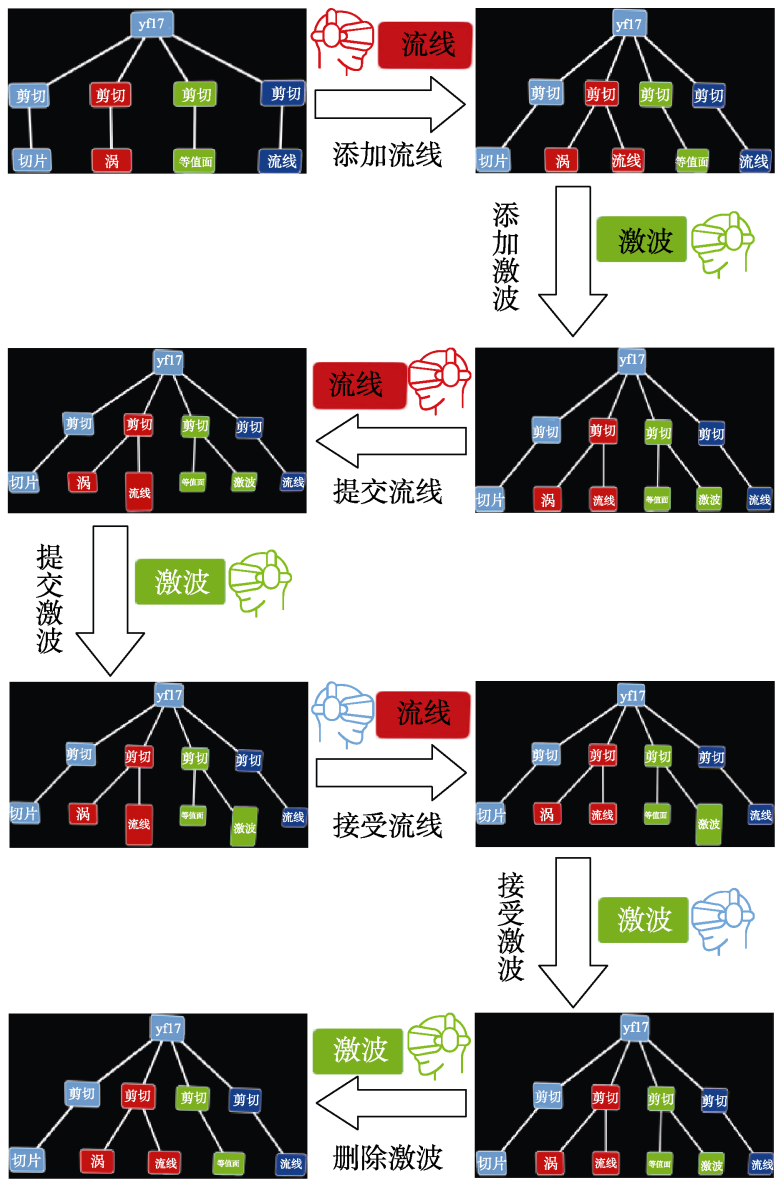

图 4 协作管线树变化过程

\subsection{4 演示协作分析}

在流场团队协作分析中，需要团队人员针对 数据中的困难问题集体攻坚, 提出各自的见解, 有 时问题难度可能会超过团队解决问题的能力, 需
要专家参与协作，指导团队克服难题，完成协作分 析. 因此，本文提出了演示协作分析模式，在沉浸 式虚拟现实协作环境中，以特权用户为核心进行 交互式可视化呈现，针对特定问题或者数据区域 开展研究分析，其余成员作为普通用户在周围观 察其可视分析过程和结果, 由此理解特权用户的 研究意图.

为了使协作团队中成员有条不紊地提出见解, 同时使普通用户能够直观地观察特权用户进行数 据交互时选择可视化方式和配置参数的过程, 更 好地理解特权用户的研究意图, 提出了以下方法.

（1）权限管理. 当团队中有专家时，默认专家 为特权用户，优先提出对问题的解决思路. 同时, 支持特权用户的身份在所有团队成员中可以任意 切换，以保证每个用户可以提交见解.

（2）视点共享. 通过将特权用户在虚拟界面上 进行交互的凝视视点位置映射到普通用户虚拟界 面对应位置，提供特权用户在虚拟界面上的具体 交互位置，以实现虚拟界面交互过程的实时共享.

\section{2 .5 自由协作分析}

流场分析是一个自由探索的过程, 不同领域 的研究人员对流场数据关注的物理量和空间位置 不同. 在团队协作中, 有的研究人员需要与其他人 相互讨论; 有的研究人员希望独自尝试, 不希望被 干扰，当有新发现时再及时共享给其他人. 因此, 本文提出了自由协作分析模式, 在共享沉浸式虚 拟现实协作环境中，所有用户均是特权用户，享有 各自独立的私人分析空间以及同一个共享空间, 允许用户对各自感兴趣的区域在私人分析空间进 行分析，并将完成的结果通过协作管线树提交至 共享空间以供团队分析. 除此以外，也可在共享空 间中直接进行共享分析.

在此模式中，存在多个特权用户同时与环境 中的配置控件和协作管线树交互时的并发冲突问 题. 针对上述问题, 本文提出冲突控制方法, 为每 个用户分别设置一组配置控件, 绑定用户 ID, 仅 允许 ID 相同的用户交互. 协作管线树上节点索引 按照创建时间顺序通过服务器维护, 并且为节点 绑定对应的用户索引, 这样其他用户就只能与属 于自己的配置控件和可视化算法节点交互, 解决 了并发冲突问题.

\subsection{6 协作消息}

在沉浸式虚拟现实流场协作中，用户之间需 要进行数据结果、配置控件、虚拟界面、虚拟化身 的同步以及权限交换信息的传递. 为了降低流场 
协作延迟并减少网络流量, 在协作通信中传递的 不是流场数据和物体空间位置, 而是能够表示虚 拟现实环境变化特征的消息, 数据渲染和计算由 客户端解析协作消息在本地完成. 消息是沉浸式 虚拟现实协作的基本通信单元，根据消息的内容 和作用的不同，可分为以下 2 类.

（1）房间消息. 用于协作房间的创建和反馈协 作房间的协作模式和流场数据信息.

(2) 交互消息. 将用户与数据、配置控件、虚 拟界面进行交互的信息, 以及用户虚拟化身和权 限交换信息统一编码为交互消息, 其结构如图 5 所 示. 交互类型表示用户执行的具体事件(如添加流 线可视化算法节点操作); 交互参数集是指执行对 应事件改变的参数(如流线种子点数量、位置和方 向), 不同的交互类型有不同的参数集; 房间 ID 对 应用户的协作房间; 交互者表示执行交互操作的 用户。

\begin{tabular}{|l|l|l|l|}
\hline 交互类型 & 交互参数集 & 房间 ID & 交互者 \\
\hline
\end{tabular}

图 5 交互消息

\section{3 本文框架实现}

\section{1 演示协作}

所有用户处于同一个共享沉浸式虚拟现实协 作环境，团队成员身份分为特权用户和普通用户， 特权用户在任何时刻至多一位, 普通用户数量不 受限制, 特权用户与普通用户之间身份可以转换. 在流场协作分析过程中，特权用户通过手势和凝 视交互方式进行数据分析时, 其虚拟参数界面、观 察视点、虚拟化身和配置特定流场可视化算法(如 流线、剪切等)的交互控件状态信息都与普通用户 共享, 保证特权用户在进行数据分析的过程对普 通用户的可见性, 以便更好地理解特权用户的分 析方法和结论. 普通用户在沉浸式虚拟现实环境 中通过行走和空间导航交互调整位置, 当视线被 遮挡时允许隐藏虚拟化身和移动虚拟界面, 以获 取合适的观察角度, 清楚地观察整个可视分析过 程. 特权用户与普通用户之间权限管理和视点共 享的实现方法如下.

（1）权限管理方法. 如图 6 所示, 在协作过程 中, 每个普通用户都能申请权限, 特权用户可以主 动释放权限让普通用户去竞争或把权限赋予给指 定用户。当协作环境中存在特权用户时, 普通用户 的申请权限信息将传给特权用户，此时协作环境
中该普通用户的虚拟化身会不断闪炼，同时特权 用户的权限管理界面会有消息反馈, 以提示特权 用户有普通用户申请权限的行为发生, 由特权用 户自行决定是否同意申请; 当协作环境中不存在 特权用户时, 若有 2 位及以上的普通用户申请权 限, 则按照申请时序赋予权限将普通用户转变为 特权用户。

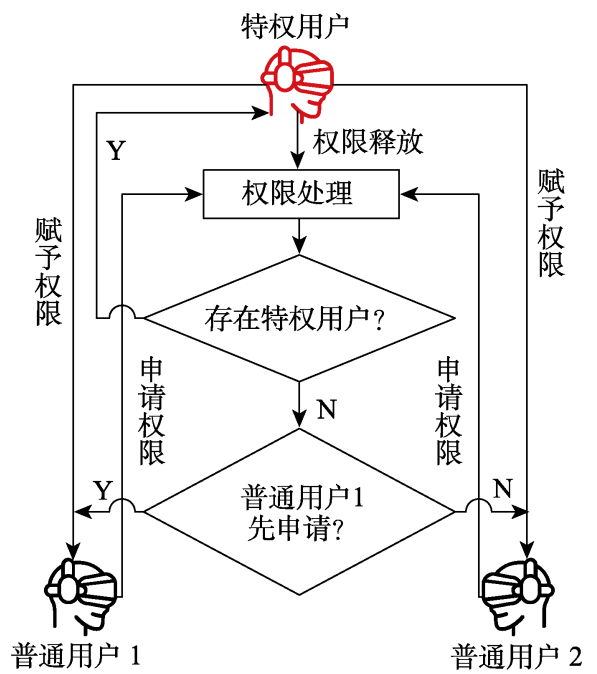

图 6 权限管理

（2）视点共享方法. 由于特权用户和普通用户 虚拟界面的空间位置不相同, 因此, 特权用户共享 视点到普通用户虚拟界面上需要进行空间坐标位 置变换, 如图 7 所示.

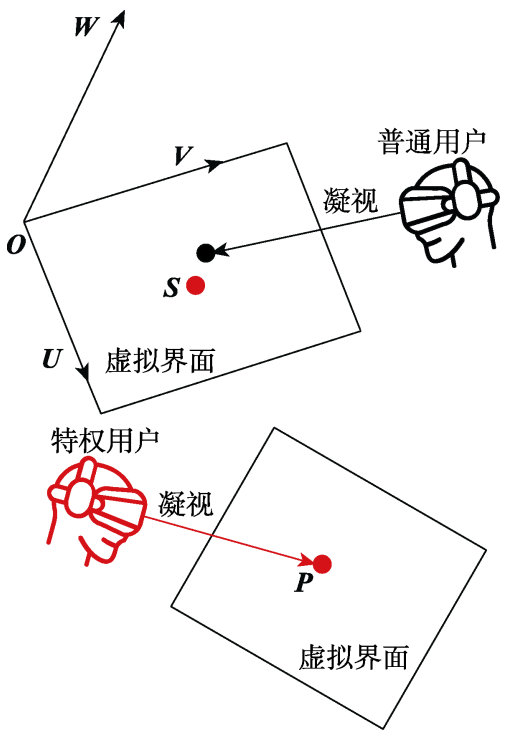

图 7 视点共享示意图

设共享视点为 $\boldsymbol{S}(x, y, z)$, 已知在特权用户虚拟 界面上的凝视视点 $\boldsymbol{P}\left(p_{1}, p_{2}, p_{3}\right)$, 普通用户虚拟界 
面坐标原点 $\boldsymbol{O}\left(o_{1}, o_{2}, o_{3}\right)$ 和坐标轴 $\boldsymbol{U}, \boldsymbol{V}, \boldsymbol{W}$ 的法向量 $\boldsymbol{U}\left(u_{1}, u_{2}, u_{3}\right), \boldsymbol{V}\left(v_{1}, v_{2}, v_{3}\right), \boldsymbol{W}\left(w_{1}, w_{2}, w_{3}\right)$. 由此得到 矩阵

$$
\boldsymbol{A}=\left[\begin{array}{rrrr}
u_{1} & u_{2} & u_{3} & -u_{1} o_{1}-u_{2} o_{2}-u_{3} o_{3} \\
w_{1} & w_{2} & w_{3} & -w_{1} o_{1}-w_{2} o_{2}-w_{3} o_{3} \\
v_{1} & v_{2} & v_{3} & -v_{1} o_{1}-v_{2} o_{2}-v_{3} o_{3} \\
0 & 0 & 0 & 1
\end{array}\right]
$$

由矩阵 $\boldsymbol{A}$ 和 $\boldsymbol{P}\left(p_{1}, p_{2}, p_{3}\right)$ 可求得在普通用户虚拟 界面上的共享视点

$$
\boldsymbol{S}(x, y, z)=\left[\begin{array}{c}
x \\
y \\
z \\
1
\end{array}\right]=\boldsymbol{A}^{-1}\left[\begin{array}{c}
p_{1} \\
p_{2} \\
p_{3} \\
1
\end{array}\right]
$$

\section{2 自由协作}

在自由协作模式中，所有用户均为特权用户. 在共享虚拟现实环境中可以自由提交需要共享的 可视化算法结果，协作管线树对应的可视化算法 节点大小改变以提示用户节点处于共享状态，由 用户自行决定是否要接受提交的内容, 避免在可 视化算法结果绘制时干扰其他用户可视分析的过 程. 同时，用户的虚拟化身、配置控件和交互操作 都是彼此可见的. 另外，在私人虚拟现实环境中， 允许用户通过协作管线树提交任意需要共享的可 视化算法节点到共享虚拟现实环境中，但用户的 虚拟化身、配置控件和交互操作彼此不可见. 用户 之间的冲突控制的实现方法如下.

如图 8 所示, 当 2 个用户同时与环境中虚拟对 象交互并产生冲突时，根据冲突的对象是协作管 线树还是配置控件分别处理.

当对象是配置控件时，仅允许用户与属于自

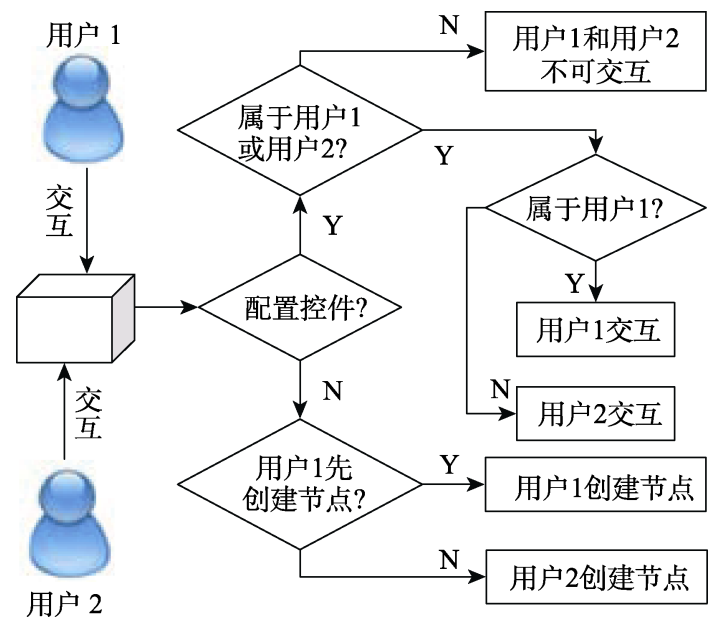

图 8 冲突控制
己的配置控件交互.

当对象是协作管线树，且用户执行创建节点 交互时，根据交互发生的时间次序，先执行交互的 用户先创建算法节点，以保证协作管线树节点的 唯一性.

\section{4 应用案例}

本文框架采用 VTK，QT，Socket，LeapMotion SDK 和 SteamVR 软件库开发, 使用 HMD 绘制沉 浸式虚拟现实场景, LeapMotion 实时采集用户手势, 采用沉浸式三维虚拟显示和多通道交互技术 ${ }^{[19]}$ 构 建原型系统, 通过多个用户对 yf17 流场数据分别 进行了演示协作分析和自由协作分析.

\section{1 演示协作案例}

在演示协作分析模式房间中，特权用户在飞 机侧翼和尾翼演示了流线可视化操作，图 9 和图 10 所示分别为特权用户和普通用户 1 对流场数据 进行分析的视图. 在图 9a 中, 特权用户通过凝视 视点在虚拟界面上进行流场算法参数配置; 在图 10a 中，普通用户 1 通过在虚拟界面上的共享视点 的位置可以观察特权用户流场算法参数的配置过 程. 图 $9 \mathrm{~b}$ 和图 $10 \mathrm{~b}$ 分别为特权用户和普通用户 1 在流线算法可视化结果共享时的视图. 在图 $9 \mathrm{~b}$ 中, 特权用户流线算法参数配置完成后，在飞机的相 应位置绘制出流线; 在图 10b 中，普通用户 1 的飞
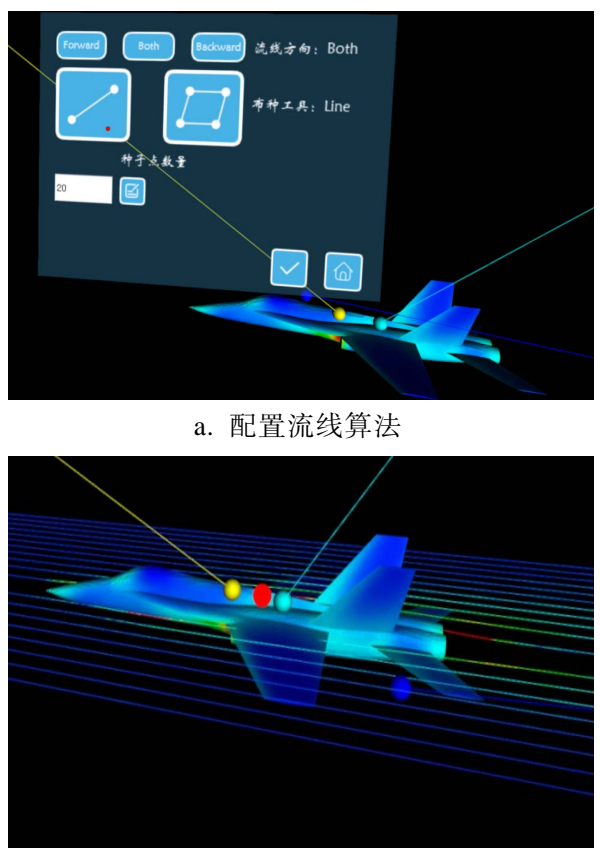

b. 流线算法结果

图 9 特权用户视图展示 
机周围绘制的流线结果与特权用户的相同，呈现 出数据的同步变化. 在沉浸式虚拟现实环境中, 通 过演示协作分析, 普通用户能直接观察到特权用 户数据分析的过程和结果, 从而进一步了解特权 用户提出的见解, 增强对流场数据中蕴含的物理 规律和现象的理解.

\section{2 自由协作案例}

在自由协作分析模式房间中，特权用户 1 和特 权用户 2 在共享虚拟现实环境中选择不同的流场 可视化算法进行数据分析, 特权用户 1 在数据节点 上添加了流线和等值面算法节点, 特权用户 2 在数 据节点上添加了剪切和切片算法, 协作管线树上 同步显示各自添加的可视化算法节点. 在图 11a 中，特权用户 1 使用线型配置控件进行流线种子点 布置. 在图 11b 中, 特权用户 2 使用盒型配置控件 确定剪切区域，用户与配置控件交互的过程彼此 可见. 在图 $11 \mathrm{c}$ 和图 $11 \mathrm{~d}$ 中, 特权用户 1 和特权用 户 2 在完成算法参数配置后, 分别共享了流线算法 节点和剪切算法节点，在协作管线树上的流线和 剪切节点宽度增加, 表示处于共享状态，允许特权

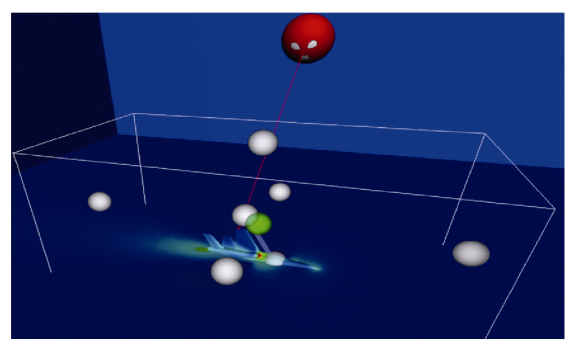

a. 特权用户 1 布置种子点

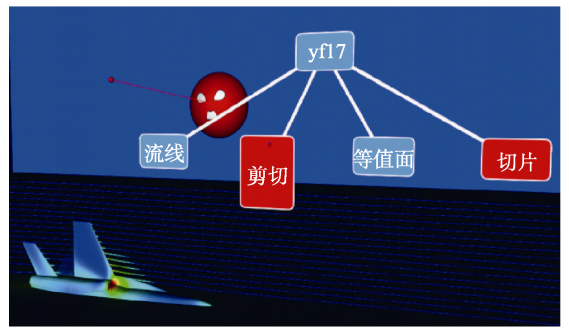

c. 特权用户 1 提交流线结果
用户 1 和特权用户 2 选择接受或拒绝彼此的共享节 点. 在图 12a 和图 12b 中, 特权用户 1 和特权用户 2 分别接受了剪切算法节点和流线算法节点, 在沉

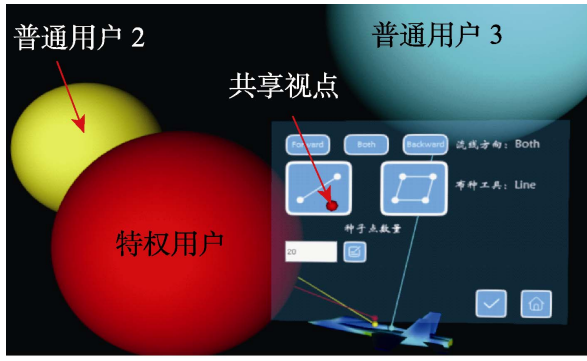

a. 配置流线算法

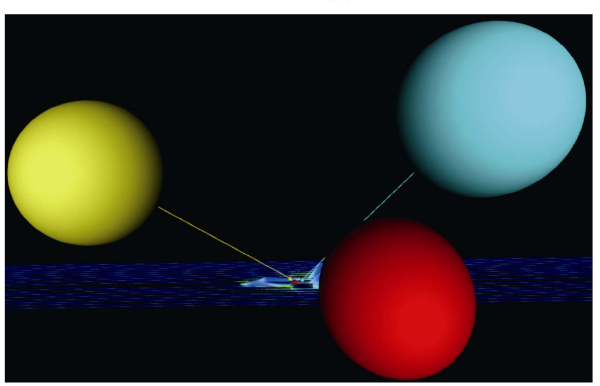

b. 流线算法结果

图 10 普通用户 1 视图展示

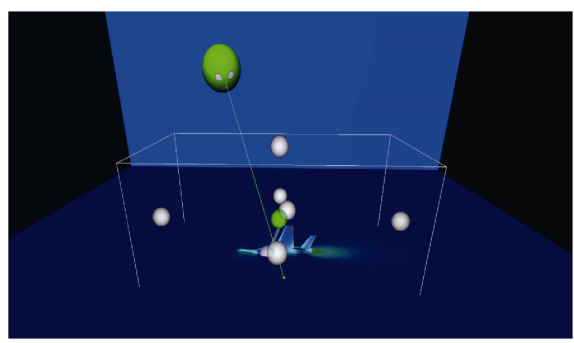

b. 特权用户2布置剪切控件

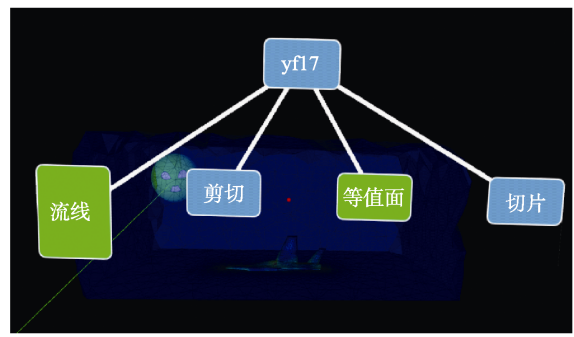

d. 特权用户 2 提交剪切结果

图 11 特权用户 1 和特权用户 2 独立分析视图展示

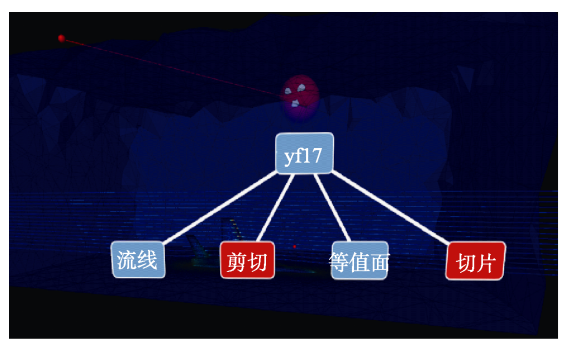

a. 特权用户1接受剪切节点

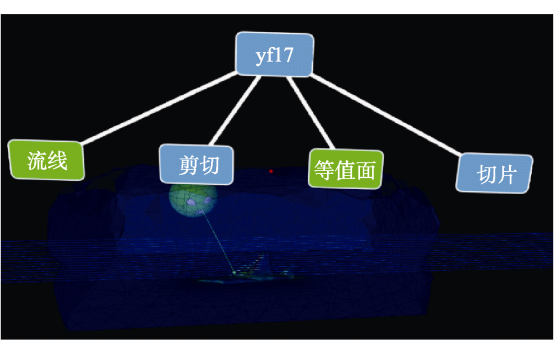

b. 特权用户 2 接受流线结果

图 12 特权用户 1 和特权用户 2 分析结果共享视图展示 
浸式虚拟现实环境中呈现出剪切节点和流线节点 的可视化结果，同时协作管线树上的剪切节点和 流线节点的高度恢复初始大小. 在自由协作分析 过程中，通过多用户自由共享的方式可以提高对整 个流场数据分析的效率, 增强对流场数据分析结果 的准确性，以更好地理解流场内部的流动机理.

\section{5 结 语}

本文设计并实现了面向流场可视化的沉浸式 虚拟现实协作框架; 构建了在沉浸式虚拟现实环 境中用户场景同步的协作消息，在基于可视化算 法树的可视化网络管理方法的基础上，提出了支 持多用户进行可视化算法节点协作共享的协作管 线树; 设计了满足流场多人协作需求的演示协作 分析模式和自由协作分析模式; 最后通过具体的 协作分析案例展示了 2 种协作分析模式.

未来还可以针对以下问题进行研究.

(1) 设计能够提高多人协作效率的工具.

（2）根据领域专家的意见，设计更多适用于流 场协作的协作分析模式.

\section{参考文献(References):}

[1] Hill G W. Group versus individual performance: are $N+1$ heads better than one?[J]. Psychological Bulletin, 1982, 91(3): 517-539

[2] van Dam A, Forsberg A S, Laidlaw D H, et al. Immersive VR for scientific visualization: a progress report[J]. IEEE Computer Graphics and Applications, 2000, 20(6): 26-52

[3] Moser I, Chiquet S, Strahm S K, et al. Group decision-making in multi-user immersive virtual reality[J]. Cyberpsychology, Behavior, and Social Networking, 2020, 23(12): 846-853

[4] Donalek C, Djorgovski S G, Cioc A, et al. Immersive and collaborative data visualization using virtual reality platforms $[\mathrm{C}]$ //Proceedings of the IEEE International Conference on Big Data. Los Alamitos: IEEE Computer Society Press, 2014: 609-614

[5] Lee B, Hu X Y, Cordeil M, et al. Shared surfaces and spaces: collaborative data visualisation in a co-located immersive environment[J]. IEEE Transactions on Visualization and Computer Graphics, 2021, 27(2): 1171-1181

[6] Capece N, Erra U, Losasso G, et al. Design and implementation of a web-based collaborative authoring tool for the virtual reality[C] //Proceedings of the 15th International Conference on Signal-Image Technology \& Internet-Based Systems. Los Alamitos: IEEE Computer Society Press, 2019: 603-610

[7] Xu Shijian, Zhao Dan, Su Chengyu, et al. Research on immersive virtual reality interactive system for visualization of flow field[J/OL]. Journal of System Simulation: 1-13[2021-07-06].
http://kns.cnki.net/kcms/detail/11.3092.V.20210408.1708.006.h tml(in Chinese)

(许世健，赵丹，苏铖宇，等. 面向流场可视化的沉浸式虚拟 现实交互系统研究[J/OL]. 系统仿真学报: 1-13[2021-07-06]. http://kns.cnki.net/kcms/detail/11.3092.V.20210408.1708.006.h tml)

[8] Ogi T, Yamada T, Hirose M, et al. Collaborative flow field visualization in the networked virtual laboratory[OL]. [2021-0706]. http://lab.sdm.keio.ac.jp/ogi/papers/IPT2004paper.pdf

[9] Febretti A, Nishimoto A, Thigpen T, et al. CAVE2: a hybrid reality environment for immersive simulation and information analysis[C] //Proceedings of the Engineering Reality of Virtual Reality. Bellingham: Society of Photo-Optical Instrumentation Engineers, 2013, 8649: 864903

[10] Hibbard B, Santek D. The VIS-5D system for easy interactive visualization[C] //Proceedings of the 1st IEEE Conference on Visualization. Los Alamitos: IEEE Computer Society Press, 1990: 28-35

[11] Bryson S, Levit C. The virtual wind tunnel[J]. IEEE Computer Graphics and Applications, 1992, 12(4): 25-34

[12] Wesche G. Three-dimensional visualization of fluid dynamics on the Responsive Workbench[J]. Future generation computer systems, 1999, 15(4): 469-475

[13] Kageyama A, Tamura Y, Sato T. Visualization of vector field by virtual reality[J]. Progress of Theoretical Physics Supplement, 2000, 138: 665-673

[14] Wasfy T M, Noor A K. Visualization of CFD results in immersive virtual environments[J]. Advances in Engineering Software, 2001, 32(9): 717-730

[15] Shetty N, Chaudhary A, Coming D, et al. Immersive ParaView: a community-based, immersive, universal scientific visualization application[C] //Proceedings of the IEEE Virtual Reality Conference. Los Alamitos: IEEE Computer Society Press, 2011: 239-240

[16] Dhillon R, Green M A. Three dimensional flow visualization in virtual reality[C] //Proceedings of the 23rd AIAA Computational Fluid Dynamics Conference. Reston: American Institute of Aeronautics and Astronautics Press, 2017: 3807

[17] O’Leary P, Jhaveri S, Chaudhary A, et al. Enhancements to VTK enabling scientific visualization in immersive environments[C] //Proceedings of the IEEE Virtual Reality. Los Alamitos: IEEE Computer Society Press, 2017: 186-194

[18] Lu S F, Cai L, Gao F. Immersive interaction design based on perception of vector field climate data[J]. Systems Science \& Control Engineering, 2019, 7(1): 116-124

[19] Chen Cheng, Zhao Dan, Wang Yueqing, et al. NNW-TopViz visual analysis system for flow fields[J]. Acta Aeronautica et Astronautica Sinica, 2021, 42(9): 246-257(in Chinese) (陈呈, 赵丹, 王岳青, 等. NNW-TopViz 流场可视分析系统 [J]. 航空学报, 2021, 42(9): 246-257)

[20] Wheless G H, Lascara C M, Leigh J, et al. CAVE6D: a tool for collaborative immersive visualization of environmental data[C] //Proceedings of the IEEE Visualization. Los Alamitos: IEEE Computer Society Press, 1998: 1-4

[21] Imai T, Johnson A E, Leigh J, et al. The virtual mail system[C] 
//Proceedings of Virtual Reality. Los Alamitos: IEEE Computer Society Press, 1999: 78

[22] Jackson R L, Fagan E. Collaboration and learning within immersive virtual reality[C] //Proceedings of the 3rd International Conference on Collaborative Virtual Environments. New York: ACM Press, 2000: 83-92

[23] Kaiser P K, Henning J G, Cotesta L, et al. Innovations in mine planning and design utilizing collaborative immersive virtual reality(CIRV)[C] //Proceedings of the 104th CIM Annual General Meeting. Montreal: Canadian Institute of Mining and Metallurgy Press, 2002: 1-7

[24] Kockro R A, Stadie A, Schwandt E, et al. A collaborative virtual reality environment for neurosurgical planning and training[J]. Operative Neurosurgery, 2007, 61(suppl_5): ONSE379ONSE391

[25] Sharma S, Jerripothula S, Mackey S, et al. Immersive virtual reality environment of a subway evacuation on a cloud for dis- aster preparedness and response training[C] //Proceedings of the IEEE Symposium on Computational Intelligence for $\mathrm{Hu}-$ man-Like Intelligence. Los Alamitos: IEEE Computer Society Press, 2014: 1-6

[26] Cordeil M, Dwyer T, Klein K, et al. Immersive collaborative analysis of network connectivity: CAVE-style or head-mounted display?[J]. IEEE Transactions on Visualization and Computer Graphics, 2017, 23(1): 441-450

[27] Mendes D, Medeiros D, Sousa M, et al. Mid-air modeling with Boolean operations in VR[C] //Proceedings of the IEEE Symposium on 3D User Interfaces. Los Alamitos: IEEE Computer Society Press, 2017: 154-157

[28] Medeiros D, dos Anjos R K, Mendes D, et al. Keep my head on my shoulders! Why third-person is bad for navigation in VR[C] //Proceedings of the 24th ACM Symposium on Virtual Reality Software and Technology. New York: ACM Press, 2018: Article No.16 